\title{
The ATLAS Tile Calorimeter Phase-II Upgrade Demonstrator Data Acquisition and Software
}

\author{
Jared Little*, on behalf of the ATLAS Collaboration \\ The University of Texas at Arlington \\ E-mail: jared.david.littledcern.ch
}

\begin{abstract}
The Phase-II upgrades will prepare the ATLAS experiment for the High Luminosity LHC (HLLHC), planned to begin operation in 2026. The HL-LHC is expected to deliver more than ten times the integrated luminosity of LHC Runs 1-3 combined. To achieve this in a reasonable amount of time, an increase in instantaneous luminosity corresponding to up to 200 simultaneous $p p$ interactions per bunch crossing is required. This large luminosisty increase presents significant challenges to the detector, trigger, and data acquisition systems in the form of increased trigger rates and detector occupancy. The results from the tests with beam performed at CERN, as well as the latest results on the development of the on- and off-detector electronics, firmware, data processing, and simulation components of the Tile Calorimeter Demonstrator readout system are presented.
\end{abstract}

Sixth Annual Conference on Large Hadron Collider Physics (LHCP2018)

4-9 June 2018

Bologna, Italy

${ }^{*}$ Speaker. 


\section{Introduction}

The Tile Calorimeter (TileCal) [2] is the central section of the hadronic calorimeter of the ATLAS experiment [1] at the Large Hadron Collider (LHC). It captures approximately $30 \%$ of jet energy and plays a crucial role in the measurement of jet- and missing-energy, jet substructure, electron isolation, and triggering. TileCal is a sampling calorimeter constructed of steel and plastic scintillator plates read out by wavelength-shifting fibres and 9852 photomultiplier tubes (PMT). The PMT signals are digitized with a $40 \mathrm{MHz}$ clock synchronous with the beam crossing.

The LHC is planning on a series of upgrades, culminating with the High-Luminosity LHC (HL-LHC) which is expected to deliver 5-7 times the current instantaneous luminosity. TileCal will undergo an upgrade to accommodate the new HL-LHC parameters. The read-out electronics will be redesigned, introducing a new strategy [3]. The full set of data generated in the detector will be digitized and sent off-detector using the PreProcessors (PPr) for every bunch crossing before any selection is applied [Figure 1]. The TileCal PPr will represent the main interface between the data acquisition, trigger, and control systems and the on-detector electronics. It will implement pipeline memories to cope with the latencies and rates specified in the new trigger schema and provide preprocessed digital trigger information to the ATLAS Level 0 trigger.

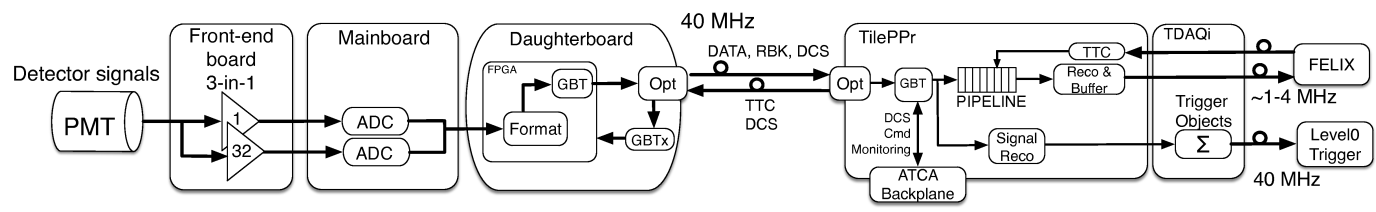

Figure 1: Phase-II TileCal front-end schematic [3].

\section{The Tile Calorimeter Demonstrator Status}

The Demonstrator Prototypes were built to evaluate the new read-out electronics while maintaining backwards compatibility with the current setup. The demonstrator has been tested in various test-beam campaigns.

The 3-in-1 Front-End Board (FEB) option has been chosen to acquire the PMT signals. These are connected to a Mainboard that provides voltage and controls, digitizes the signal and sends it to the Daughterboard. The Daughterboard is the interface between the on- and off-detector electronics.

The PreProcessor is the core element of the back-end system [Figure 2], providing communication with the front-end to transmit commands and to receive the digitized PMT data. Once a trigger signal is received, the data is formatted and transmitted to the legacy Read-Out Driver to maintain compatibility. During the HL-LHC the Trigger and DAQ interface (TDAQi) will provide preprocessed information to the new trigger and Front-End Link eXchange (FELIX) systems, the core of the new ATLAS Trigger/DAQ architecture. 


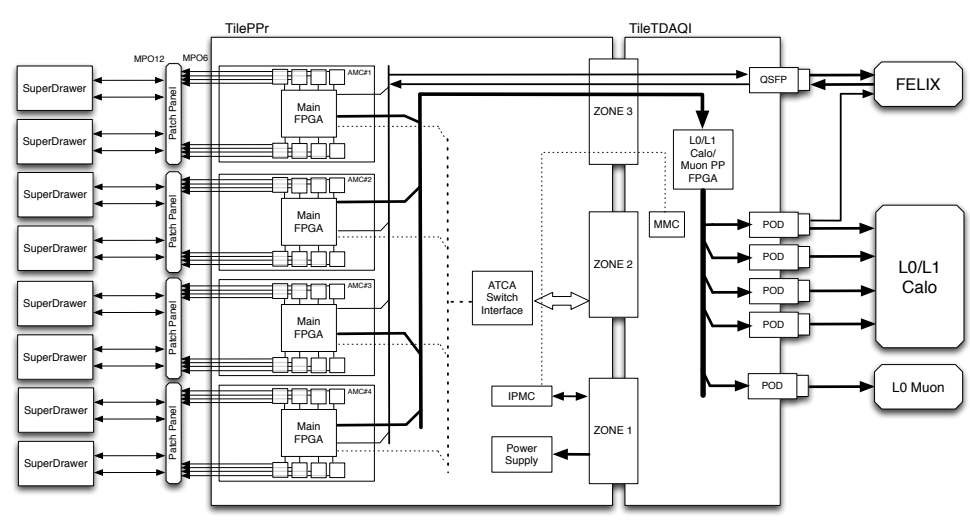

Figure 2: Block diagram of the final PPr design [3].

\section{Test Beam}

Extensive beam tests have been performed with the final electronics designs planned for the TileCal upgrade for HL-LHC. Three TileCal modules were exposed to different particles with different energies and incident angles. The beams were produced by extracting $E_{\text {beam }}=400 \mathrm{GeV}$ protons from the Super Proton Synchrotron machine and producing secondary and tertiary beams by placing beryllium and polyethylene in the path. These beams are expected to be composed mostly of pions and protons, but electrons, muons, and kaons are also present. Results obtained using experimental data are compared to simulation obtained using the GEANT4 toolkit [4].

The demonstrator was inserted into a long barrel module and equipped with 3-in-1 cards. The data selected by the trigger system is stored in raw data files through the legacy Read-Out System (ROS) and the new FELIX system in parallel. The ROS sends the data packets and saves the detector and beam data in a local disk. The PPr prototype [Figure 3] is the primary component of the off-detector electronics. It reads out the demonstrator and transmits the selected data to the legacy ROD and FELIX systems.

The ATLAS software framework (Athena) is used to reconstruct the raw data stored in the Event Builder and FELIX to energy and time per cell. This operation is performed online for some events for monitoring.
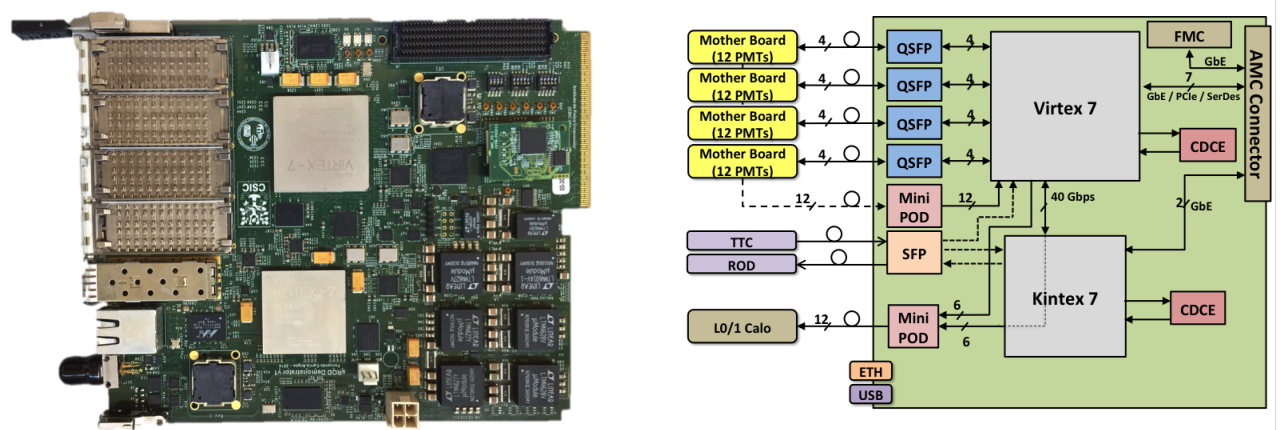

Figure 3: Picture (left) and block diagram (right) of the PPr Demonstrator module [3]. 


\section{Test Beam Results}

The interaction of muons with matter is well understood. The dominant energy loss process is ionisation and the amount of energy loss is essentially proportional to the muon track length. The data was taken with $E_{\text {beam }}=165 \mathrm{GeV}$ muon beams incident at 90 degrees.

The response was studied as the ratio between the energy deposited in a cell (dE) over the track length (dl). In Figure 4 (left), the experimental and simulated distributions of $d E / d l$ in cell A8 are shown. In Figure 4 (right), the Data/MC value for all of the A-cells are displayed. A uniformity of $1 \%$ and a maximum offset of $4 \%$ are observed.
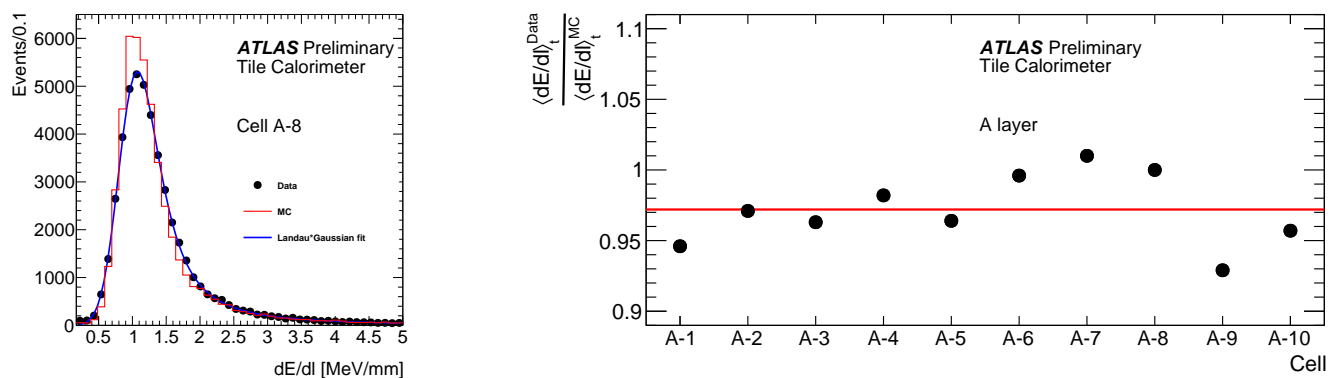

Figure 4: Deposited energy per unit length for cell A-8 shown for data and simulation (left) and comparison for Data/MC for A-layer (right) [3].

The responses of $E_{\text {beam }}=20,50$, and $100 \mathrm{GeV}$ electron beams incident on the center of cell A-4 at 20 degrees were measured. It is expected that electrons deposit all of their energy in the calorimeter, and since the layers were already calibrated at the EM scale we expect that the energy read-out divided by the beam energy to be one. Results are presented in Figure 5.

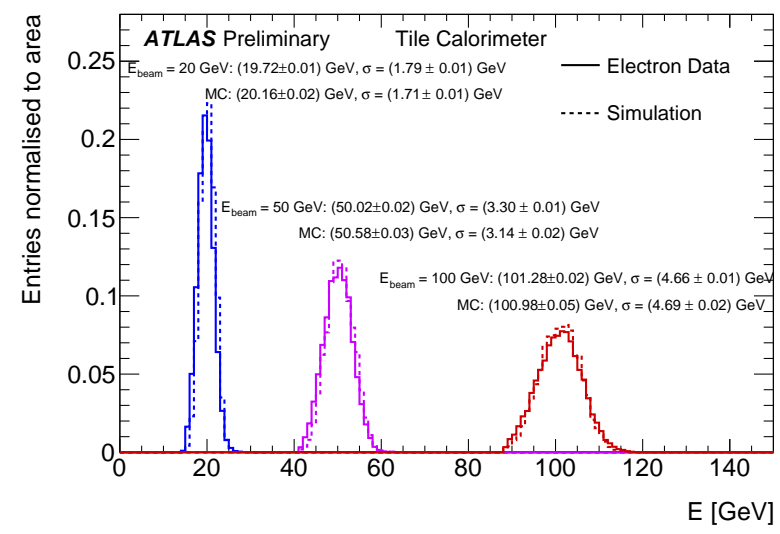

Figure 5: Electron data compared to simulation, for $E_{\text {beam }}=20,50$, and $100 \mathrm{GeV}$ [3].

The role of the hadronic calorimeter in ATLAS is to measure the energies of jets. Presented here are a few results showing the response for pions, kaons, and protons for different beam energies. The largest fraction of the shower energy is deposited in the cells of the demonstrator module. Particles are identified using the calorimeter response and three beam line Cherenkov counters. Electron contamination is reduced by a thin lead absorber along the beam line. 
Response is calculated using the mean of a Gaussian fit performed within $\pm 2 \sigma$ of the peak as shown in Figure 6 (left). In Figure 6 (right) the results for kaons, protons, and pions compared with simulation are shown. The mean value of the fitted Gaussian is the reported value. The expected linearity of the response as a function of beam energy was observed.
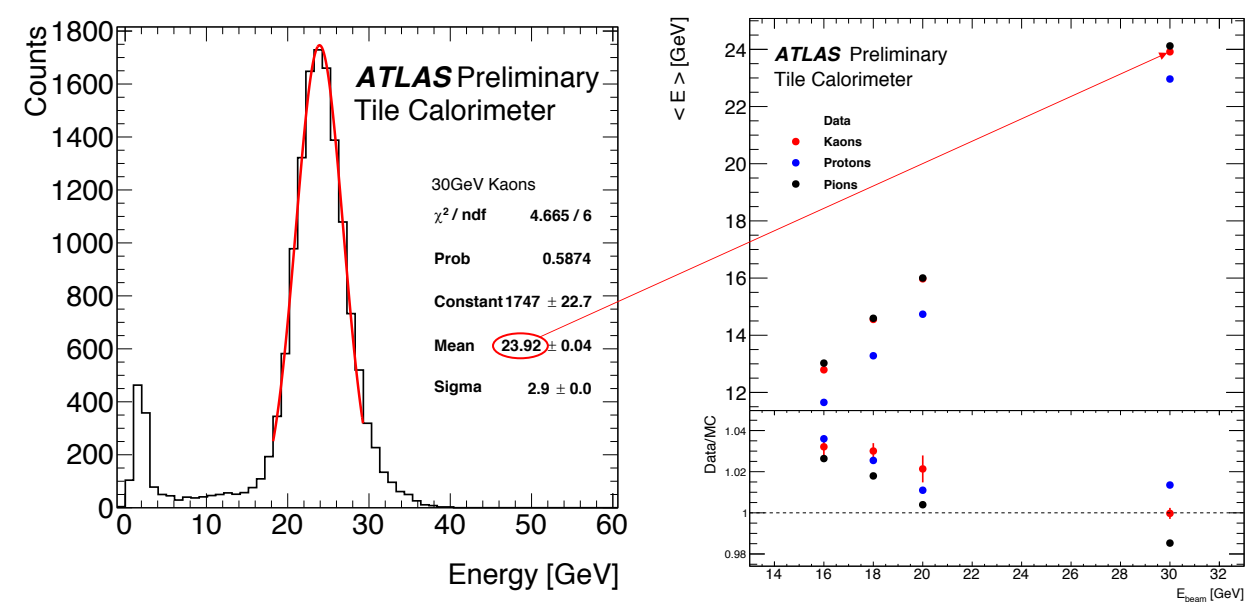

Figure 6: Kaon/Proton/Pion energy extracted and filled for multiple beam energies [3].

\section{Conclusion}

Research and development is ongoing for the TileCal Phase-II upgrades. Demonstrator prototypes have been produced and tested with beam while maintaining backwards compatibility, with the goal of insertion into ATLAS for more thorough testing. All of the prototypes performed well during the tests with beam, measuring well the response to electrons, muons, and hadrons. The technical design report [3] has been approved and printed.

\section{References}

[1] ATLAS Collaboration,, The ATLAS Experiment at the CERN Large Hadron Collider, JINST 3 (2008) S08003.

[2] ATLAS Collaboration, ATLAS Tile Calorimeter: Technical Design Report, Tech. Rep. CERN-LHCC-96-042; ATLAS-TDR-3, CERN, Geneva, Dec, 1996.

https://cds.cern.ch/record/331062.

[3] ATLAS Collaboration, Technical Design Report for the Phase-II Upgrade of the ATLAS Tile Calorimeter, Tech. Rep. CERN-LHCC-2017-019; ATLAS-TDR-028, CERN, Geneva, Sep, 2017. https://cds.cern.ch/record/2285583.

[4] GEANT4 Collaboration, S. Agostinelli et al., GEANT4: A Simulation toolkit, Nucl. Instrum. Meth. A506 (2003) 250-303.

Supported by the H2020 project AIDA-2020, GA no. 654168 